\title{
Quasi-Coordinate Search for a Randomly Moving Target
}

\author{
A. A. M. Teamah ${ }^{1}$, W. A. Afifi ${ }^{2,3^{*}}$ \\ ${ }^{1}$ Department of Mathematics, Faculty of Science, Tanta University, Tanta, Egypt \\ ${ }^{2}$ Mathematics and Statistics Department, College of Science, Taibah University, Yanbu, KSA \\ ${ }^{3}$ Mathematics and Statistics Department, Canal Higher Institute of Engineering and Technology, Suez, Egypt \\ Email: *Walaa_affy@yahoo.com
}

How to cite this paper: Teamah, A.A.M. and Afifi, W.A. (2019) Quasi-Coordinate Search for a Randomly Moving Target. Journal of Applied Mathematics and Physics, 7, 1814-1825.

https://doi.org/10.4236/jamp.2019.78124

Received: July 8, 2019

Accepted: August 16, 2019

Published: August 19, 2019

Copyright (c) 2019 by author(s) and Scientific Research Publishing Inc. This work is licensed under the Creative Commons Attribution International License (CC BY 4.0).

http://creativecommons.org/licenses/by/4.0/

\begin{abstract}
In this paper, we study the quasi-coordinated search technique for a lost target assumed to move randomly on one of two disjoint lines according to a random walk motion, where there are two searchers beginning their search from the origin on the first line and other two searchers begin their search from the origin on the second line. But the motion of the two searchers on the first line is independent from the motion of the other two searchers on the second line. Here we introduce a model of search plan and investigate the expected value of the first meeting time between one of the searchers and the lost target. Also, we prove the existence of a search plan which minimizes the expected value of the first meeting time between one of the searchers and the target.
\end{abstract}

\section{Keywords}

Random Walker, Linear Search, Expected Value, Optimal Search Plane, Stochastic Process

\section{Introduction}

The searching for a lost target either located or moved is often a time-critical issue, that is, when the target is very important. The primary objective is to find and search for the lost target as soon as possible. The searching for lost targets has recently applications such as the search for a goldmine underground, the search for Landmines and navy mines, the search for the cancer cells in the human body, the search for missing black box of a plane crash in the depth of the sea of ocean, the search for a damaged unit in a large linear system such as telephone lines, and mining system, and so on [1] [2] [3]. Search problem when the 
lost target is located or moved on the real line has been considered in [4]-[9]. The coordinated search technique discussed on the real line when the located target has symmetric or unsymmetric distribution as in [10] [11] [12]. Also, the coordinated search for a located target in the plane has been examined in [13] [14] [15] [16]. Recently, [17] and [18] proposed and studied a modern search model in the three-dimensional space to find a 3-D randomly located target by one searcher, two searchers and four searchers.

\section{Problem Formulations}

One of the most complicate problems when a mother loses her son in a way of multiple ways, here the primary objective is finding the lost son, as soon as possible in a minimum time. The survival rate of the son in this region gradually decreases, so the search team must organize itself quickly to begin the mission of the searching for the lost son immediately. Also, when the target is serious as a car, which filled by explosives, and it moves on one road from disjoint roads, and then the search effort must be unrestricted and we can use more than searcher to detect the target at right time.

The search team which consists of 4 searchers will organize itself on 2 straight lines to find the lost target as soon as possible. We clarify a modern technique by collaboration between each two searchers to find the lost person in minimum time. This problem can be characterized as follows.

\subsection{The Searching Framework}

The space of search: 2 disjoint lines.

The target: The target moves with a random walk motion on one of 2 disjoint straight lines.

The means of search: Looking for the lost target performed by two searches on each line. The searchers start searching for the target from the origins of the two lines with continuous paths and with equal speeds. In addition, the search spaces (2 straight lines) are separated into many distances.

\subsection{The Searching Technique}

Assume that we have two searchers $S_{1}$ and $S_{2}$ that start together looking for the lost target from $O_{1}$ on $L_{1}$. The two searchers coordinate their search about the lost target, where the searcher $S_{1}$ searches to the right and goes from the $O_{1}$ to $H_{1}$, and the searcher $S_{2}$ searches to the left and goes from $O_{1}$ to $-H_{1}$, the two searchers $S_{1}$ and $S_{2}$ reach to $H_{1}$ and $-H_{1}$ in the same time of $G_{1}$. Then they come back to $O_{1}$ again in the same time of $G_{2}$. If one of the two searchers do not find the lost target, then the two searchers $S_{1}$ and $S_{2}$ begin the new cycle search for the lost target, where they go from $\mathrm{O}_{1}$ to $H_{2}$ and $-H_{2}$, respectively and they will reach to $H_{2}$ and $-H_{2}$ in the same time of $G_{3}$. Then they come back to $O_{1}$ again in the same time of $G_{4}$ and so on. Also, we have two other searchers $S_{3}$ and $S_{4}$ start together looking for the lost target from $O_{2}$ on the second line $L_{2}$, the searcher $S_{3}$ 
searchers to the right and goes from $O_{2}$ to $\bar{H}_{1}$, and the searchers $S_{4}$ searches to the left and goes to the left and goes from $O_{2}$ to $-\bar{H}_{1}$, the two searchers $S_{3}$ and $S_{4}$ reach to $\bar{H}_{1}$ and $-\bar{H}_{1}$ in the same time of $\bar{G}_{1}$. Then they come back to $O_{2}$ again in the same time of $\bar{G}_{2}$. If one of the two searchers not find the lost target, then the two searchers $S_{3}$ and $S_{4}$ begin the new cycle search for the lost target, where they go from $\mathrm{O}_{2}$ to $\bar{H}_{2}$ and $-\bar{H}_{2}$, respectively and they will reach to $\bar{H}_{2}$ and $-\bar{H}_{2}$ in the same time of $\bar{G}_{3}$, then they come back to $O_{2}$ again in the same time of $\bar{G}_{4}$, and so on. The four searchers return to the $O_{1}$ and $O_{2}$ after searching successively common distances until the target is found.

\subsection{The Movement of the Target and the Searchers}

A target is assumed to move randomly on one of two disjoint lines according to a stochastic process $\left\{S(t), t \in I^{+}\right\}, I^{+}=\{0,1,2, \cdots\}$. Assume that $\left\{Z_{i}\right\}_{i \geq 0}$ is a sequence of independent identically distributed random variables such as for any $i \geq 1: \quad p\left(Z_{i}=1\right)=p$ and $p\left(Z_{i}=-1\right)=1-p=q$, where $p, q>0$. For $t>0$, $t \in I^{+}$,

$$
S(t)=\sum_{i=1}^{t} Z_{i}, S(0)=0
$$

We assume the searchers $S_{1}$ and $S_{2}$ begin their search path from $O_{1}$ on $L_{1}$ with speeds $V_{1}$, and the searchers $S_{3}$ and $S_{4}$ begin their search path from $O_{2}$ on $L_{2}$ with speeds $V_{2}$, following the search paths which are functions $\phi_{1}: R^{+} \rightarrow R$ and $\bar{\phi}_{1}: R^{+} \rightarrow R$ on $L_{1}$ and $\phi_{2}: R^{+} \rightarrow R$ and $\bar{\phi}_{2}: R^{+} \rightarrow R$ on $L_{2}$, respectively, such that:

$$
\left|\phi_{1}\left(t_{1}\right)-\phi_{1}\left(t_{2}\right)\right|=\left|\bar{\phi}_{1}\left(t_{1}\right)-\bar{\phi}_{1}\left(t_{2}\right)\right| \leq V_{1}\left|t_{1}-t_{2}\right|
$$

and

$$
\left|\phi_{2}\left(t_{1}\right)-\phi_{2}\left(t_{2}\right)\right|=\left|\bar{\phi}_{2}\left(t_{1}\right)-\bar{\phi}_{2}\left(t_{2}\right)\right| \leq V_{2}\left|t_{1}-t_{2}\right|, \forall t_{1}, t_{2} \in I^{+},
$$

where $V_{1}$ and $V_{2}$ are constants in $R^{+}$and $\phi_{1}(0)=\bar{\phi}_{1}(0)=\phi_{2}(0)=\bar{\phi}_{2}(0)=0$. Let the set of all search paths of the two searchers $S_{1}$ and $S_{2}$, which satisfy condition (1), be respectively by $\Phi_{v_{1}}$ and $\bar{\Phi}_{v_{1}}$ respectively and the set of all search paths of the searchers $S_{3}$ and $S_{4}$ which satisfy condition (2), be represented by $\Phi_{v_{2}}$ and $\bar{\Phi}_{v_{2}}$, respectively. we represented to the path of $S_{1}$ and $S_{2}$ by $\phi_{0}=\left(\phi_{1}, \bar{\phi}_{1}\right) \in \Phi_{0}$ where $\bar{\phi}_{0}=\left(\phi_{2}, \bar{\phi}_{2}\right) \in \bar{\Phi}_{0}$, where

$$
\bar{\Phi}_{0}=\left\{\left(\phi_{2}, \bar{\phi}_{2}\right): \phi_{2} \in \Phi_{v_{2}}, \bar{\phi}_{2} \in \bar{\Phi}_{v_{2}}\right\} .
$$

The search plan of the four searchers be represented by $\hat{\phi}=\left(\phi_{0}, \bar{\phi}_{0}\right) \in \hat{\Phi}$, where $\hat{\Phi}=\left\{\left(\phi_{0}, \bar{\phi}_{0}\right): \phi_{0} \in \Phi_{0}, \bar{\phi}_{0} \in \bar{\Phi}_{0}\right\}$ is the set of all search plan.

We assume that $Z_{0}=X$ if the target moves on $L_{1}$ and $Z_{0}=Y$ if the target moves on $L_{2}$ such that $P\left(Z_{0}=X\right)+P\left(Z_{0}=Y\right)=1$. There is a known probability measure $v_{1}+v_{2}=1$ on $L_{1} \cup L_{2}$ which describes the location of the target, where $v_{1}$ is probability measure induced by the position of the target on $L_{1}$, while $V_{2}$ on $L_{2}$. The first meeting time valued in $I^{+}$defined as 


$$
\begin{array}{r}
\tau_{\hat{\phi}}=\inf \left\{t: \phi_{1}(t)=X+S(t) \text { or } \bar{\phi}_{1}(t)=X+S(t)\right. \\
\text { or } \left.\phi_{2}(t)=Y+S(t) \text { or } \bar{\phi}_{2}(t)=Y+S(t)\right\},
\end{array}
$$

where $Z_{0}$ is a random variable representing the initial position of the target and valued in $2 I$ (or $2 I+1)$ and independent of $S(t), t>0$.

At the beginning of the search suppose that the lost target is existing on any integer point on $L_{1}$ but more than $H_{1}$ or less than $-H_{1}$ or the lost target is existing on an integer point on $L_{2}$ but more than $\bar{H}_{1}$ or less than $-\bar{H}_{1}$. Let $\tau_{\phi_{1}}$ be the first meeting time between $S_{1}$ and the target and $\tau_{\hat{\phi}_{1}}$ be the first meeting time between $S_{2}$ and the target and $\tau_{\phi_{2}}$ be the first meeting time between $S_{3}$ and the target and $\tau_{\hat{\phi}_{2}}$ be the first meeting time between $S_{4}$ and the target. The main objective is to find the search plan $\hat{\phi}=\left(\phi_{0}, \bar{\phi}_{0}\right) \in \hat{\Phi}$ such that $E\left(\tau_{\hat{\phi}}\right)<\infty$. In this case $\hat{\phi}$ is said to be a finite search plan, and if $E\left(\tau_{\hat{\phi}}^{*}\right)<E\left(\tau_{\hat{\phi}}\right), \forall \hat{\phi} \in \hat{\Phi}$, where $E$ terms to expectation value, then we call $\hat{\phi}^{*}$ is an optimal search plan. Given $n>0$, if $z$ is: $0 \leq k_{1} \leq \frac{n+z}{2} \leq n$, where $k_{1}$ is integer, then

$$
p\left(S(n)=k_{1}\right)=\left\{\begin{array}{l}
\left(\begin{array}{l}
n \\
k_{1}
\end{array}\right) p^{k_{1}} q^{n-k_{1}} \\
0, \text { if } k_{1} \text { does not exist }
\end{array}\right.
$$

\subsection{Finite Search Plan}

Let $\lambda_{1}, \lambda_{2}, \zeta_{1}, \zeta_{2}$ be positive integers such that $\zeta_{1}, \zeta_{2}>1, \lambda_{1}=k \theta_{1}, \lambda_{2}=k \theta_{2}$, where $k=1,2, \cdots$ and $\theta_{1}, \theta_{2}$ are the least positive integers and $V_{1}=V_{2}=1$.

We shall define the sequences $\left\{G_{i}\right\}_{i \geq 0},\left\{H_{i}\right\}_{i \geq 0}$ for the searcher $S_{1}$ on the first line $L_{1}$ and $\left\{\bar{G}_{i}\right\}_{i \geq 0},\left\{\bar{H}_{i}\right\}_{i \geq 0}$ for the searcher $S_{3}$ on the second line $L_{2}$ and the search plans with speeds 1 as follows:

$$
\begin{aligned}
& G_{i}=2^{\frac{1}{2}\left[1-(-1)^{i-1}\right]} \lambda_{1}\left(\zeta_{1}^{\frac{i}{2}+\frac{1}{4}-(-1)^{i} \frac{1}{4}}-1\right), H_{i}=G_{2 i-1}, i \geq 1 \text { on } L_{1}, \\
& \bar{G}_{i}=2^{\frac{1}{2}\left[1-(-1)^{i-1}\right]} \lambda_{2}\left(\zeta_{2}^{\frac{i}{2}^{2}+\frac{1}{4}-(-1)^{i} \frac{1}{4}}-1\right), \bar{H}_{i}=\bar{G}_{2 i-1}, i \geq 1 \text { on } L_{2} .
\end{aligned}
$$

We shall define the search path as follows:

for any $t \in I^{+}$, if $G_{i} \leq t<G_{i+1}$, then

$$
\phi_{1}(t)=\left(\frac{1}{2} H_{\frac{i+1}{2}}\right)+(-1)^{i+1}\left(\frac{1}{2} H_{\frac{i+1}{2}}\right)+(-1)^{i}\left(t-G_{i}\right),
$$

and

$$
\bar{\phi}_{1}(t)=-\phi_{1}(t) .
$$

Also, if $\bar{G}_{i} \leq t<\bar{G}_{i+1}$, then

$$
\phi_{2}(t)=\left(\frac{1}{2} \bar{H}_{\frac{i+1}{2}}\right)+(-1)^{i+1}\left(\frac{1}{2} \bar{H}_{\frac{i+1}{2}}\right)+(-1)^{i}\left(t-\bar{G}_{i}\right),
$$

and

$$
\bar{\phi}_{2}(t)=-\phi_{2}(t) .
$$


We define the notion

$$
\begin{aligned}
& \varphi_{1}(t)=S(t)-t, \tilde{\varphi}_{1}(t)=S(t)+t \text { on } L_{1}, \\
& \varphi_{2}(t)=S(t)-t, \tilde{\varphi}_{2}(t)=S(t)+t \text { on } L_{2},
\end{aligned}
$$

the searchers $S_{1}$ and $S_{2}$ return to the origin of $L_{1}$ after searching successively common distances $H_{1}, H_{2}, H_{3}, \cdots$, and $-H_{1},-H_{2},-H_{3}, \cdots$, respectively and the searchers $S_{3}$ and $S_{4}$ return to the origin of $L_{2}$ after searching successively common distances $\bar{H}_{1}, \bar{H}_{2}, \bar{H}_{3}, \cdots$, and $-\bar{H}_{1},-\bar{H}_{2},-\bar{H}_{3}, \cdots$, respectively until the target is found.

Theorem 1: If $\hat{\phi}=\left(\phi_{0}, \bar{\phi}_{0}\right) \in \hat{\Phi}$ is a search plan defined above, then the expectation $E\left(\tau_{\hat{\phi}}\right)$ if finite if

$$
\begin{gathered}
w_{1}(x)=\sum_{i=1}^{\infty}\left(\zeta_{1}^{i}-1\right) p\left(\tilde{\varphi}_{1}\left(G_{2 i-1}\right)<-x\right), \\
w_{2}(x)=\sum_{i=1}^{\infty}\left(\zeta_{1}^{i}-1\right) p\left(\varphi_{1}\left(G_{2 i-1}\right)>-x\right), \\
w_{3}(x)=\sum_{i=1}^{\infty}\left(\zeta_{1}^{i}\left(\zeta_{1}^{i}-2\right)+1\right) p\left(\tilde{\varphi}_{1}\left(G_{2 i}\right)<-x\right), \\
w_{4}(y)=\sum_{i=1}^{\infty}\left(\zeta_{1}^{i}\left(\zeta_{1}^{i}-2\right)+1\right) p\left(\varphi_{1}\left(G_{2 i}\right)>-x\right), \\
w_{5}(y)=\sum_{i=1}^{\infty}\left(\zeta_{2}^{i}-1\right) p\left(\tilde{\varphi}_{2}\left(G_{2 i-1}\right)<-y\right), \\
w_{6}(y)=\sum_{i=1}^{\infty}\left(\zeta_{2}^{i}-1\right) p\left(\varphi_{2}\left(\tilde{G}_{2 i-1}\right)>-y\right), \\
w_{7}(y)=\sum_{i=1}^{\infty}\left(\zeta_{2}^{i}\left(\zeta_{2}^{i}-2\right)+1\right) p\left(\tilde{\varphi}_{2}\left(\tilde{G}_{2 i}\right)<-y\right),
\end{gathered}
$$

and

$$
w_{8}(y)=\sum_{i=1}^{\infty}\left(\zeta_{2}^{i}\left(\zeta_{2}^{i}-2\right)+1\right) p\left(\varphi_{2}\left(\tilde{G}_{2 i}\right)>-y\right)
$$

are finite.

Proof: Assume that $X$ and $Y$ are independent of $S(t), t>0$, if $X>0$, then $X+S(t)>\phi_{1}(t)$ until the first meeting between $S_{1}$ and the target on $L_{1}$, also if $X<0$, then $X+S(t)<\hat{\phi}_{1}(t)$ until the first meeting between $S_{2}$ and the target on $L_{2}$. We can apply this assumption on the second line by replacing $X$ by $Y$ and $\phi_{1}, \bar{\phi}_{1}$ by $\phi_{2}, \bar{\phi}_{2}$ respectively. Hence, for any $i \geq 0$

$$
p\left(\tau_{\hat{\phi}}>t\right)=p\left(\tau_{\phi_{0}}>t \text { or } \tau_{\hat{\phi}_{0}}>t\right),
$$

hence

$$
\begin{aligned}
E\left(\tau_{\hat{\phi}}\right) & =\int_{0}^{\infty} p\left(\tau_{\hat{\phi}}>t\right) \mathrm{d} t \\
& \leq \sum_{i=0}^{\infty}\left[\int_{G_{i}}^{G_{i+1}} p\left(\tau_{\phi_{0}}>G_{i}\right) \mathrm{d} t+\int_{\tilde{G}_{i}}^{\tilde{G}_{i+1}} p\left(\tau_{\hat{\phi}_{0}}>\tilde{G}_{i}\right) \mathrm{d} t\right] \\
& =\sum_{i=0}^{\infty}\left(2^{\frac{1}{2}\left[1-(-1)^{i+2}\right]} \lambda_{1}\left(\zeta_{1}^{\frac{i+1}{2}+\frac{1}{4}-(-1)^{i+1} \frac{1}{4}}-1\right)\right.
\end{aligned}
$$




$$
\begin{aligned}
& \left.-2^{\frac{1}{2}\left[1-(-1)^{i+1}\right]} \lambda_{1}\left(\zeta_{1}^{\frac{i}{2}+\frac{1}{4}-(-1)^{i} \frac{1}{4}}-1\right)\right) p\left(\tau_{\hat{\phi}_{0}}>G_{i}\right) \\
& +\left(2^{\frac{1}{2}\left[1-(-1)^{i+2}\right]} \lambda_{2}\left(\zeta_{2}^{\frac{i+1}{2}+\frac{1}{4}-(-1)^{i+1} \frac{1}{4}}-1\right)\right. \\
& \left.-2^{\frac{1}{2}\left[1-(-1)^{i+1}\right]} \lambda_{2}\left(\zeta_{2}^{\frac{i}{2}+\frac{1}{4}-(-1)^{\frac{1}{4}}}-1\right)\right) p\left(\tau_{\hat{\phi}_{0}}>\tilde{G}_{i}\right) \\
& =\lambda_{1}\left[\left(\left(\zeta_{1}-2\right)+1\right) p\left(\tau_{\phi_{0}}>0\right)+\left(\zeta_{1}-1\right) p\left(\tau_{\phi_{0}}>G_{1}\right)\right. \\
& +\left(\zeta_{1}\left(\zeta_{1}-2\right)+1\right) p\left(\tau_{\phi_{0}}>G_{2}\right)+\left(\zeta_{1}^{2}-1\right) p\left(\tau_{\phi_{0}}>G_{3}\right) \\
& +\left(\zeta_{1}^{2}\left(\zeta_{1}-2\right)+1\right) p\left(\tau_{\phi_{0}}>G_{4}\right)+\left(\zeta_{1}^{3}-1\right) p\left(\tau_{\phi_{0}}>G_{5}\right) \\
& \left.+\left(\zeta_{1}^{3}\left(\zeta_{1}-2\right)+1\right) p\left(\tau_{\phi_{0}}>G_{6}\right)+\cdots\right] \\
& +\lambda_{2}\left[\left(\left(\zeta_{2}-2\right)+1\right) p\left(\tau_{\overline{\phi_{0}}}>0\right)+\left(\zeta_{2}-1\right) p\left(\tau_{\bar{\phi}_{0}}>\bar{G}_{1}\right)\right. \\
& +\left(\zeta_{2}\left(\zeta_{2}-2\right)+1\right) p\left(\tau_{\overline{\phi_{0}}}>\bar{G}_{2}\right)+\left(\zeta_{2}^{2}-1\right) p\left(\tau_{\overline{\phi_{0}}}>\bar{G}_{3}\right) \\
& +\left(\zeta_{2}^{2}\left(\zeta_{2}-2\right)+1\right) p\left(\tau_{\bar{\phi}_{0}}>\bar{G}_{4}\right)+\left(\zeta_{2}^{3}-1\right) p\left(\tau_{\overline{\phi_{0}}}>\bar{G}_{5}\right) \\
& \left.+\left(\zeta_{2}^{3}\left(\zeta_{2}-2\right)+1\right) p\left(\tau_{\overline{\phi_{0}}}>\bar{G}_{6}\right)+\cdots\right]
\end{aligned}
$$

to solve Equation (4) we shall find the value of $p\left(\tau_{\phi_{0}}>G_{2 i-1}\right), p\left(\tau_{\bar{\phi}_{0}}>\bar{G}_{2 i-1}\right)$, $p\left(\tau_{\phi_{0}}>G_{2 i}\right)$ and the value of $p\left(\tau_{\bar{\phi}_{0}}>\bar{G}_{2 i}\right)$ as the following

$$
\begin{aligned}
p\left(\tau_{\phi_{0}}>G_{2 i-1}\right) \leq & \int_{-\infty}^{0} p\left(x+S\left(G_{2 i-1}\right)<-H_{i} / X=x\right) v_{1}(\mathrm{~d} x) \\
& +\int_{0}^{\infty} p\left(x+S\left(G_{2 i-1}\right)>H_{i} / X=x\right) v_{1}(\mathrm{~d} x)
\end{aligned}
$$

We get

$$
\begin{aligned}
p\left(\tau_{\phi_{0}}>G_{2 i-1}\right) \leq & \int_{-\infty}^{0} p\left(\tilde{\varphi}_{1}\left(G_{2 i-1}\right)<-x\right) v_{1}(\mathrm{~d} x) \\
& +\int_{0}^{\infty} p\left(\varphi_{1}\left(G_{2 i-1}\right)>-x\right) v_{1}(\mathrm{~d} x)
\end{aligned}
$$

also,

$$
\begin{aligned}
p\left(\tau_{\bar{\phi}_{0}}>\bar{G}_{2 i-1}\right) \leq & \int_{-\infty}^{0} p\left(\tilde{\varphi}_{2}\left(\bar{G}_{2 i-1}\right)<-y\right) v_{2}(\mathrm{~d} y) \\
& +\int_{0}^{\infty} p\left(\varphi_{2}\left(\bar{G}_{2 i-1}\right)>-y\right) v_{2}(\mathrm{~d} y) \\
p\left(\tau_{\phi_{0}}>G_{2 i}\right) \leq & \int_{-\infty}^{0} p\left(X+S\left(G_{2 i}\right)<-2 H_{i}\right) v_{1}(\mathrm{~d} x) \\
& +\int_{0}^{\infty} p\left(x+S\left(G_{2 i}\right)>2 H_{i}\right) v_{1}(\mathrm{~d} x)
\end{aligned}
$$

We get

$$
p\left(\tau_{\phi_{0}}>G_{2 i}\right) \leq \int_{-\infty}^{0} p\left(\tilde{\varphi}_{1}\left(G_{2 i}\right)<-x\right) v_{1}(\mathrm{~d} x)+\int_{0}^{\infty} p\left(\varphi_{1}\left(G_{2 i}\right)>-x\right) v_{1}(\mathrm{~d} x)
$$




$$
p\left(\tau_{\phi_{0}}>\bar{G}_{2 i}\right) \leq \int_{-\infty}^{0} p\left(\tilde{\varphi}_{1}\left(\bar{G}_{2 i}\right)<-y\right) v_{2}(\mathrm{~d} y)+\int_{0}^{\infty} p\left(\varphi_{1}\left(\bar{G}_{2 i}\right)>-y\right) v_{2}(\mathrm{~d} y)
$$

substituting by (5), (6), (7) and (8) in (4) we can get

$$
\begin{aligned}
E\left(\tau_{\hat{\phi}}\right) \leq & \lambda_{1}\left[\left(\left(\zeta_{1}-2\right)+1\right) p\left(\tau_{\phi_{0}}>0\right)+\left(\zeta_{1}-1\right) p\left(\tau_{\phi_{0}}>G_{1}\right)\right. \\
& +\left(\zeta_{1}\left(\zeta_{1}-2\right)+1\right) p\left(\tau_{\phi_{0}}>G_{2}\right)+\left(\zeta_{1}^{2}-1\right) p\left(\tau_{\phi_{0}}>G_{3}\right) \\
& +\left(\zeta_{1}^{2}\left(\zeta_{1}-2\right)+1\right) p\left(\tau_{\phi_{0}}>G_{4}\right)+\left(\zeta_{1}^{3}-1\right) p\left(\tau_{\phi_{0}}>G_{5}\right) \\
& \left.+\left(\zeta_{1}^{3}\left(\zeta_{1}-2\right)+1\right) p\left(\tau_{\phi_{0}}>G_{6}\right)+\cdots\right] \\
+ & \lambda_{2}\left[\left(\left(\zeta_{2}-2\right)+1\right) p\left(\tau_{\overline{\phi_{0}}}>0\right)+\left(\zeta_{2}-1\right) p\left(\tau_{\overline{\phi_{0}}}>\bar{G}_{1}\right)\right. \\
+ & \left(\zeta_{2}\left(\zeta_{2}-2\right)+1\right) p\left(\tau_{\bar{\phi}_{0}}>\bar{G}_{2}\right)+\left(\zeta_{2}^{2}-1\right) p\left(\tau_{\bar{\phi}_{0}}>\bar{G}_{3}\right) \\
+ & \left(\zeta_{2}^{2}\left(\zeta_{2}-2\right)+1\right) p\left(\tau_{\bar{\phi}_{0}}>\bar{G}_{4}\right)+\left(\zeta_{2}^{3}-1\right) p\left(\tau_{\overline{\phi_{0}}}>\bar{G}_{5}\right) \\
+ & \left.\left(\zeta_{2}^{3}\left(\zeta_{2}-2\right)+1\right) p\left(\tau_{\bar{\phi}_{0}}>\bar{G}_{6}\right)+\cdots\right]
\end{aligned}
$$

hence

$$
\begin{aligned}
E\left(\tau_{\hat{\phi}}\right) \leq & \lambda_{1}\left[\left(\left(\zeta_{1}-2\right)+1\right) p\left(\tau_{\phi_{0}}>0\right)+\left\{\int_{-\infty}^{0} w_{1}(x) v_{1}(\mathrm{~d} x)\right.\right. \\
& \left.\left.+\int_{0}^{\infty} w_{2}(x) v_{1}(\mathrm{~d} x)\right\}+\left\{\int_{-\infty}^{0} w_{3}(y) v_{2}(\mathrm{~d} y)+\int_{0}^{\infty} w_{4}(y) v_{2}(\mathrm{~d} y)\right\}\right] \\
& +\lambda_{2}\left[\left(\left(\zeta_{2}-2\right)+1\right) p\left(\tau_{\overline{\phi_{0}}}>0\right)+\left\{\int_{-\infty}^{0} w_{5}(y) v_{2}(\mathrm{~d} y)\right.\right. \\
& \left.\left.+\int_{0}^{\infty} w_{6}(y) v_{2}(\mathrm{~d} y)\right\}+\left\{\int_{-\infty}^{0} w_{7}(y) v_{2}(\mathrm{~d} y)+\int_{0}^{\infty} w_{8}(y) v_{2}(\mathrm{~d} y)\right\}\right]
\end{aligned}
$$

where,

$$
\begin{gathered}
w_{1}(x)=\sum_{i=1}^{\infty}\left(\zeta_{1}^{i}-1\right) p\left(\tilde{\varphi}_{1}\left(G_{2 i-1}\right)<-x\right), \\
w_{2}(x)=\sum_{i=1}^{\infty}\left(\zeta_{1}^{i}-1\right) p\left(\varphi_{1}\left(G_{2 i-1}\right)>-x\right), \\
w_{3}(x)=\sum_{i=1}^{\infty}\left(\zeta_{1}^{i}\left(\zeta_{1}-2\right)+1\right) p\left(\tilde{\varphi}_{1}\left(G_{2 i}\right)<-x\right), \\
w_{4}(x)=\sum_{i=1}^{\infty}\left(\zeta_{1}^{i}\left(\zeta_{1}-2\right)+1\right) p\left(\varphi_{1}\left(G_{2 i}\right)>-x\right), \\
w_{5}(y)=\sum_{i=1}^{\infty}\left(\zeta_{2}^{i}-1\right) p\left(\tilde{\varphi}_{2}\left(\bar{G}_{2 i-1}\right)<-y\right), \\
w_{6}(y)=\sum_{i=1}^{\infty}\left(\zeta_{2}^{i}-1\right) p\left(\varphi_{2}\left(\bar{G}_{2 i-1}\right)>-y\right), \\
w_{7}(y)=\sum_{i=1}^{\infty}\left(\zeta_{2}^{i}\left(\zeta_{2}-2\right)+1\right) p\left(\tilde{\varphi}_{2}\left(\bar{G}_{2 i}\right)<-y\right),
\end{gathered}
$$

and 


$$
w_{8}(y)=\sum_{i=1}^{\infty}\left(\zeta_{2}^{i}\left(\zeta_{2}-2\right)+1\right) p\left(\varphi_{2}\left(\bar{G}_{2 i}\right)>-y\right) .
$$

Lemma 1: For any $k \geq 0$, let $a_{n} \geq 0$ for $n \geq 0$, and $a_{n+1} \leq a_{n}$. Let $\left\{d_{n}\right\}_{n \geq 0}$ be a strictly increasing sequence of integers with $d_{0}=0$,

$$
\sum_{n=k}^{\infty}\left(d_{n+1}-d_{n}\right) a_{d_{n+1}} \leq \sum_{k=d_{k}}^{\infty} a_{k} \leq \sum_{n=k}^{\infty}\left(d_{n+1}-d_{n}\right) a_{d_{n}}
$$

For more details see [1].

Theorem 2: The chosen search plan satisfies

$$
\begin{gathered}
w_{1}(x) \leq w_{9}(|x|), \quad w_{2}(x) \leq w_{10}(|x|), \\
w_{2}(x) \leq w_{11}(|x|), \quad w_{4}(x) \leq w_{12}(|x|), \\
w_{5}(y) \leq w_{13}(|y|), \quad w_{6}(y) \leq w_{14}(|y|), \\
w_{7}(y) \leq w_{15}(|y|), \text { and } w_{8}(y) \leq w_{16}(|y|),
\end{gathered}
$$

where, $w_{9}(|x|), \quad w_{10}(|x|), \quad w_{11}(|x|), \quad w_{12}(|x|), \quad w_{13}(|y|), \quad w_{14}(|y|), \quad w_{15}(|y|)$, and $w_{16}(|y|)$ are linear function.

Proof: This theorem will prove for $w_{2}(x)$ and $w_{6}(y)$, and by similar way we can prove the other cases

$$
w_{2}(x)=\sum_{i=0}^{\infty}\left(\zeta_{1}^{i}-1\right) p\left(\varphi_{1}\left(G_{2 i-1}\right)>-x\right)
$$

and

$$
w_{6}(y)=\sum_{i=0}^{\infty}\left(\zeta_{2}^{i}-1\right) p\left(\varphi_{2}\left(\bar{G}_{2 i-1}\right)>-y\right)
$$

1) if $x \leq 0$, then

$$
w_{2}(x) \leq w_{2}(0)
$$

and if $y \leq 0$, then

$$
w_{6}(y) \leq w_{6}(0)
$$

2) if $x>0$, then

$$
w_{2}(x)=w_{2}(0)+\sum_{i=0}^{\infty}\left(\zeta_{1}^{i}-1\right) p\left(-x<\varphi_{1}\left(G_{2 i-1}\right) \leq 0\right),
$$

and if $y>0$, then

$$
w_{6}(y)=w_{6}(0)+\sum_{i=0}^{\infty}\left(\zeta_{2}^{i}-1\right) p\left(-y<\varphi_{2}\left(\bar{G}_{2 i-1}\right) \leq 0\right),
$$

from Theorem (2), see (Mohamed [1]) we obtain

$$
w_{2}(0)=\sum_{i=0}^{\infty}\left(\zeta_{1}^{i}-1\right) p\left(\varphi_{1}\left(G_{2 i-1}\right)>0\right) \leq \sum_{i=1}^{\infty}\left(\zeta_{1}^{i}-1\right) \varepsilon^{G_{2 i-1}}, 0<\varepsilon<1
$$

and

$$
w_{6}(0)=\sum_{i=0}^{\infty}\left(\zeta_{2}^{i}-1\right) p\left(\varphi_{2}\left(\bar{G}_{2 i-1}\right)>0\right) \leq \sum_{i=1}^{\infty}\left(\zeta_{2}^{i}-1\right) \varepsilon^{\bar{G}_{2 i-1}}, 0<\varepsilon<1
$$


Let us define the following

1) $V(n)=\varphi_{1}\left(n \theta_{1}\right) / 2=\sum_{i=1}^{n} W_{i}$, where $\left\{W_{i}\right\}$ is a sequence of (i. i. d. r. v.) $\bar{V}(n)=\varphi_{2}\left(n \theta_{2}\right) / 2=\sum_{i=1}^{n} \bar{W}_{i}$, where $\left\{\bar{W}_{i}\right\}$ is a sequence of (i. i. d. r. v.).

2) $d_{n}=G_{2 n-1} / \theta_{1}=k\left(\zeta_{1}^{n}-1\right), \bar{d}_{n}=\bar{G}_{2 n-1} / \theta_{2}=k\left(\zeta_{2}^{n}-1\right)$.

3) $a(n)=\frac{n}{n+k} p(-x / 2<V(n) \leq 0)=\sum_{i=0}^{x \mid / 2} p[-(j+1)<V(n) \leq(-j)]$,

$\bar{a}(n)=\frac{n}{n+k} p(-y / 2<\bar{V}(n) \leq 0)=\sum_{i=0}^{|y| / 2} p[-(j+1)<\bar{V}(n) \leq(-j)]$,

4) $m_{1}$ is an integer such that $d m_{1}=b_{1}|x|+b_{2}$, and $m_{2}$ is an integer such that $d m_{2}=\bar{b}_{1}|y|+\bar{b}_{2}$,

5) $\alpha_{1}=\frac{\zeta_{1}}{\left(\zeta_{1}-1\right) k}$, and $\alpha_{2}=\frac{\zeta_{2}}{\left(\zeta_{2}-1\right) k}$,

and

6) $U_{1}(j, j+1)=\sum_{n=0}^{\infty} p[-(j+1)<V(n)<(-j)]$,

$\bar{U}_{1}(j, j+1)=\sum_{n=0}^{\infty} p[-(j+1)<\bar{V}(n) \leq(-j)]$,

then $U_{1}(j, j+1)$ and $\bar{U}_{1}(j, j+1)$ satisfies the condition of the renewal equation, for more details see [19].

If $n>d_{m_{1}}$ and $n>d_{m_{2}}$ then by Theorem (2) see (Mohamed [1]) $a(n)$ and $\bar{a}(n)$ are non increasing and we can apply Lemma (2) to obtain

$$
\begin{aligned}
w_{2}(x)-w_{2}(0) & =\sum_{i=1}^{\infty}\left(\zeta_{1}^{i}-1\right) p\left(-x<\varphi_{1}\left(G_{2 i-1}\right) \leq 0\right) \\
& =\sum_{n=1}^{n_{1}} \zeta_{1}^{n} a\left(d_{n}\right)+\sum_{n=n_{1}+1}^{\infty} \zeta_{1}^{n} a\left(d_{n}\right) \\
& \leq \sum_{n=1}^{n_{1}} \zeta_{1}^{n}+\alpha_{1} \sum_{n=n_{1}+1}^{\infty}\left(d_{n}-d_{n-1}\right) a\left(d_{n}\right) \\
& \leq \sum_{n=1}^{n_{1}} \zeta_{1}^{n}+\alpha_{1} \sum_{n=d_{m_{1}}}^{\infty} a(n) \\
& \leq \sum_{n=1}^{n_{1}} \zeta_{1}^{n}+\alpha_{1} \sum_{n=d_{m_{1}}}^{\infty} \sum_{i=0}^{|x| / 2} p[-(j+1)<V(n) \leq(-j)] \\
& \leq \sum_{n=1}^{n_{1}} \zeta_{1}^{n}+\alpha_{1} \sum_{j=0}^{|x| / 2} U_{1}(j, j+1)
\end{aligned}
$$

and

$$
\begin{aligned}
w_{6}(x)-w_{6}(0) & =\sum_{i=0}^{\infty}\left(\zeta_{2}^{i}-1\right) p\left(-y<\varphi_{2}\left(G_{2 i-1}\right) \leq 0\right) \\
& =\sum_{n=1}^{n_{2}} \zeta_{2}^{n} a\left(\bar{d}_{n}\right)+\sum_{n=n_{2}+1}^{\infty} \zeta_{2}^{n} a\left(\bar{d}_{n}\right) \\
& \leq \sum_{n=1}^{n_{2}} \zeta_{2}^{n}+\alpha_{2} \sum_{n=n_{2}+1}^{\infty}\left(\bar{d}_{n}-\bar{d}_{n-1}\right) a\left(\bar{d}_{n}\right)
\end{aligned}
$$




$$
\begin{aligned}
& \leq \sum_{n=1}^{n_{2}} \zeta_{2}^{n}+\alpha_{2} \sum_{n=d_{m_{2}}}^{\infty} \bar{a}(n) \\
& \leq \sum_{n=1}^{n_{2}} \zeta_{2}^{n}+\alpha_{2} \sum_{j=0}^{|y| / 2} \bar{U}_{1}(j, j+1)
\end{aligned}
$$

Since $U_{1}(j, j+1)$ and $\bar{U}_{1}(j, j+1)$ satisfied the condition of the renewal equation, hence $U_{1}(j, j+1)$ and $\bar{U}_{1}(j, j+1)$ is bounded for all $j$ by a constant, so

$$
w_{2}(x) \leq w_{2}(0)+N_{1}+N_{2}|x|=w_{10}(|x|)
$$

and

$$
w_{6}(x) \leq w_{6}(0)+\bar{N}_{1}+\bar{N}_{2}|x|=w_{14}(|y|) .
$$

Theorem 3: If $\hat{\phi}=\left(\phi_{0}, \bar{\phi}_{0}\right) \in \hat{\Phi}$ is a finite search plan, then $E\left|Z_{0}\right|$ is finite.

Proof: If $E\left(\tau_{\hat{\phi}}\right)<\infty$, then $p\left(\tau_{\hat{\phi}}\right.$ is finite $)=1$ and so

$$
p\left(\tau_{\phi_{0}} \text { is finite }\right)+p\left(\tau_{\hat{\phi}_{0}} \text { is finite }\right)=1 \text {, }
$$

then, we conclude that

$$
p\left(\tau_{\phi_{0}} \text { is finite }\right)=1 \text { and } p\left(\tau_{\overline{\phi_{0}}} \text { is finite }\right)=0,
$$

or

$$
p\left(\tau_{\phi_{0}} \text { is finite }\right)=0 \text { and } p\left(\tau_{\bar{\phi}_{0}} \text { is finite }\right)=1 .
$$

On the first line $L_{1}$ if $p\left(\tau_{\phi_{0}}\right.$ is finite $)=1$, then $X_{0}=\phi\left(\tau_{\phi_{0}}\right)-S\left(\tau_{\phi_{0}}\right)$ with probability one and hence

$$
E\left|X_{0}\right| \leq E\left(\tau_{\phi_{0}}\right)+E\left|S\left(\tau_{\phi_{0}}\right)\right| .
$$

If $E\left(\tau_{\phi_{0}}\right)<\infty$, but $\left|S\left(\tau_{\phi_{0}}\right)\right| \leq \tau_{\phi_{0}}$, then $E\left|S\left(\tau_{\phi_{0}}\right)\right| \leq E\left(\tau_{\phi_{0}}\right)$ and $E\left|X_{0}\right|<\infty$. On the second line $L_{2}$ if $p\left(\tau_{\bar{\phi}_{0}}\right.$ is finite $)=1$, then $Y_{0}=\phi\left(\tau_{\bar{\phi}_{0}}\right)-S\left(\tau_{\bar{\phi}_{0}}\right)$ with probability one, by the same way we can get $E\left|Y_{0}\right|$ is finite on the second line $L_{2}$.

\section{Existence of an Optimal Search Plan}

Theorem 4: Let for any $t \in I^{+}$, let $S(t)$ be a process. The mapping $\hat{\phi} \rightarrow E\left(\tau_{\hat{\phi}}\right) \in R^{+}$is lower semi-continuous on $\hat{\Phi}(t)$.

Proof: Let $I(\hat{\phi}, t)$ be the indicator function of the set $\left\{\tau_{\hat{\phi}} \geq t\right\}$ by the Fatou Lebesque theorem see (Stone [16]) we get

$$
\begin{aligned}
E\left(\tau_{\hat{\phi}}\right) & =E\left[\sum_{t=1}^{\infty} I(\hat{\phi}, t)\right] \\
& =E\left[\sum_{t=1}^{\infty} \liminf _{i \rightarrow \infty} I\left(\hat{\phi}_{n}, t\right)\right] \leq \liminf _{i \rightarrow \infty} E\left(\tau_{\hat{\phi}}\right),
\end{aligned}
$$

for any sequence $\hat{\phi}_{n} \rightarrow \hat{\phi}$ in $\hat{\Phi}(t)$ is sequentially compact [20], thus the mapping $\hat{\phi} \rightarrow E\left(\tau_{\hat{\phi}}\right)$ is lower semi continuous on $\hat{\Phi}(t)$, then this mapping attains its minimum. 


\section{Conclusions}

We have described a new kind of search technique to find a lost moving target on one of two disjoint lines. The motion of the four searchers on the two lines in the quasi-coordinated search technique is independent, and this helps us to find the lost target without waste of time and cost, especially if this target is valuable as the search for lost children. Actually we calculated the finite search plan. Also; we proved the existence of an optimal search plan which minimizes the expected value of the first meeting time between one of the searchers and the target.

In the future work, we will introduce an important search problem, looking for a randomly moving target as a general case and the searchers will begin their mission from any point on the line.

\section{Conflicts of Interest}

The authors declare no conflicts of interest regarding the publication of this paper.

\section{References}

[1] Mohamed, A.A. and El-Rayes, A.B. (1989) Search for a Randomly Moving Target. The Third ORMA Conference, Vol. 2, 323-329.

[2] Alpern, S. and Howard, J.V. (2000) Alternating Search at Two Locations. Dynamics and Control, 10, 319-339. https://doi.org/10.1023/A:1011245715521

[3] El-Hadidy, M.A. and El-Bagoury, A.H. (2016) Optimal Search Strategy for a Three-Dimensional Randomly Located Target. International Journal of Operational Research, 29, 115-126. http://www.inderscience.com/link.php?id=83178 https://doi.org/10.1504/IJOR.2017.10003932

[4] Mohamed, A.A. (2005) The Generalized Search for One Dimensional Random Walker. International Journal of Pure and Applied Mathematics, 19, 375-387.

[5] Mohamed, A.A. and Abou-Gabal, H.M. (2003) Linear Search with Multiple Searchers for a Randomly Moving Target. International Conference for Statistics, Computer Science and its Application, 115-124.

[6] Mohamed, A.A. and Abou-Gabal, H.M. (2004) Multiplicative Linear Search Problem. Egyptian Statistical Journal, Cairo University, 48, 34-45.

[7] Beck, A. and Warren, P. (1973) The Return of the Linear Search Problem. Israel Journal of Mathematics, 14, 169-183. https://doi.org/10.1007/BF02762672

[8] Balkhi, Z.T. (1989) The Generalized Optimal Search Paths for Continuous Univariate Random Variable. Journal of the Operations Research, 23, 67-96. https://doi.org/10.1051/ro/1989230100671

[9] Stone, L.D. (1975) Theory of Optimal Search. Academic Press, New York.

[10] Mohamed, A.A., Abou-Gabal, H.M. and Afifi, W.A. (2013) Double Coordinate Search Problem. International Journal of Contemporary Mathematical Science, 8.

[11] Mohamed, A.A., Abou-Gabal, H.M. and Afifi, W.A. (2016) Generalized Coordinated Search for a Randomly Located Target. Delta Journal of Science, 38.

[12] Reyniers, D.J. (1996) Coordinated Search for an Object on the Line. European Journal of Operational Research, 95, 663-670.

https://doi.org/10.1016/S0377-2217(96)00314-1 
[13] Mohamed, A.A. and El-Hadidy, M. (2013) Coordinated Search for a Conditionally Deterministic Target Motion in the Plan. European Journal of Mathematical Sciences, 2, 272-295.

[14] Mohamed, A.A., Abou-Gabal, H.M. and El-Hadidy, M. (2009) Coordinated Search for a Randomly Located Target on the Plane. European Journal of Pure and Applied Mathematics, 2, 97-111.

[15] Mohamed, A.A., Fergany, H.A. and El-Hadidy, M. (2012) On the Coordinated Search Problem on the Plane. Istanbul University Journal of the School of Business Administration, 41, 80-102.

[16] Bourgault, F., Furukawa, T. and Durrant-Whyte, H. (2003) Coordinated Decentralized Search for a Lost Target in a Bayesian World. Proceedings IEEERSJ International Conference, Intelligent Robots and Systems, Vol. 1.

[17] Mohamed, A.A. and EL-Bagoury, A.H. (2019) Minimizing the Expected Time to Detect a Randomly Located Lost Target Using 3-Dimensional Search Technique. Journal of Communications in Statistics.

[18] Mohamed, A.A., El-Hadidy, M. and EL-Bagoury, A.H. (2017) 3-Dimensional Coordinated Search Technique for a Randomly Located Target. International Journal of Computing Science and Mathematics, 9. https://doi.org/10.1504/IJCSM.2018.093152

[19] Feller, W. (1966) An Introduction to Probability Theory and Its Applications. Second Edition, Wiley, New York.

[20] Mohamed, A.A., El-Rayes, A.B. and Abou-Gabal, H.M. (2003) Linear Search for a Brownian Target Motion. Acta Mathematica Scientia, 23B, 321-327. https://doi.org/10.1016/S0252-9602(17)30338-7 